\title{
Análisis comparativo sobre la afectividad como motivadora del proceso enseñanza-aprendizaje - casos: Argentina, Colombia y Ecuador
}

\section{Comparative analysis on affection as a motivator of teaching-learning process - Cases: Argentine, Colombia and Ecuador}

\author{
MSc. María Elena Godoy Zúñiga \\ MSc. Bertha Janeth Campoverde Rojas \\ Facultad de Comunicación \\ Universidad de Especialidades Espíritu Santo - Samborondón \\ Ecuador. Emails: mariaegodoy@uees.edu.ec, bcampo@uees. \\ edu.ec. \\ Cómo citar: Godoy, M.E; Campoverde, B.J. (2016) Análisis \\ comparativo sobre la afectividad como motivadora del proceso \\ enseñanza-aprendizaje.Casos: Argentina,Colombia y Ecuador. \\ Sophia 12 (2): 217-231.
}

\section{Resumen}

\begin{abstract}
En este artículo se aborda el aspecto de la afectividad por parte de la docencia dentro del salón de clase en los países de Argentina, Colombia y Ecuador, desde una revisión bibliográfica. Esto es, con la finalidad de coadyuvar en la definición conceptual del problema, desde una perspectiva latinoamericana. Durante las últimas décadas, pedagogos, educadores y psicólogos han expuesto desde sus diferentes postulados, el valor de la afectividad como un requisito primordial en el proceso enseñanza- aprendizaje y la importancia que conlleva tener excelentes relaciones interpersonales entre docentes y educandos. Entre estas perspectivas se consideran algunos criterios que caracterizan el concepto de la afectividad como la motivación, la inteligencia interpersonal y la sensibilidad en el afecto que deben ser impartidas desde la cátedra, de tal manera que pueda estimular el auto concepto de los estudiantes y superar actitudes de baja autoestima, complejos de culpa, coerción y un autoritarismo en las aulas escolares.
\end{abstract}

Palabras clave: Afectividad, ambiente educacional, enseñanza-aprendizaje, motivación.

\begin{abstract}
This article approaches the subject of affection by teachers within the classroom in Argentine, Colombia and Ecuador, from a bibliographic review. The purpose is to contribute in the conceptual definition of the problem, from a Latin American perspective. During the las decades, teachers and psychologists have expressed from their various postulates, the value of affection as a primary requirement in teaching - learning process, and the importance of keeping excellent personal relationships between teachers and students. These perspectives include some criterions that characterize the concept of affection such as motivation, interpersonal intelligence and sensibility in affection that should be taught from the cathedra, in such a manner that the students' self concept may be encouraged, and overcome low self esteem attitudes, guilt, coercion and authoritarianism at the classroom.
\end{abstract}

Keywords: Affectivity, teaching-learning, motivation, educational environment. 


\section{Introducción}

Los contextos de violencia que viven muchos estudiantes en las aulas escolares han llamado la atención en la última década, siendo objeto de estudio la creación de políticas públicas y la rápida intervención dentro de estos focos de acción (Abramovay, 2005; Gázquez, Pérez-Fuentes, Carrión y Santiuste, 2010). La importancia que tiene la convivencia humana y el rechazo a la violencia en la educación, se ve reflejada en la declaración de la Unesco (2007) del período 2001-2010 como la "Década Internacional por una Cultura de Paz y No-Violencia para los Niños del Mundo", de manera que se logre comprender los aspectos que dificultan este llamado a la igualdad y asegurar el éxito de la sociedad a través de todos los involucrados en el ámbito educativo.

En lo que respecta al Ecuador, se parte del desinterés entre estudiantes y maestros ante la poca afectividad manifestada dentro del aula de clases, consecuencia de ello es la generación de un clima tenso, ocasionando, al mismo tiempo, un bajo rendimiento académico. Por otro lado, los conflictos de cualquier índole, inciden en los estudiantes quienes poseen diferentes formas de entender la realidad y de concebir el saber, a partir de su historia personal o pasado oculto. Por este motivo, todas las ponencias invitan al profesorado a comprender las angustias, los miedos, las conductas compulsivas, las dificultades de relación, los problemas de aprendizaje, los malos comportamientos, el contexto familiar, todo este cúmulo de características que, de manera a veces exacerbada, los educandos llevan a la sala escolar (Jung, 2001). Dicho esto, la afectividad dentro del campo educativo coadyuva a la creación de un clima cálido dentro del salón de clase y a la formación de un individuo más proactivo para la sociedad, ante esta problemática, resulta relevante desarrollar el tema de la afectividad como un ente vital dentro de la escolaridad latinoamericana.

Por este motivo, este artículo tiene como objetivo describir el estado actual sobre la afectividad del docente dentro de las aulas de clase en los países de Argentina, Ecuador y Colombia, con la finalidad de motivar al profesorado a la creación de ambientes cálidos dentro de la comunidad educativa.
La estructura de este trabajo inicia con los antecedentes que describen la realidad social de un clima educativo donde la afectividad desempeña un papel secundario, haciendo referencia a las consecuencias negativas que se generan ante la carencia de este factor de relevancia en las aulas. Luego se define el concepto de afectividad y se lo caracteriza vinculándolo con su uso por parte de los docentes. A continuación se presentan los conceptos de inteligencia interpersonal, sensibilidad en el afecto y motivación, como factores básicos y estratégicos que coadyuvan al surgimiento del trato cordial entre profesores y estudiantes. Con base en las vertientes indicadas se exponen casos reales suscitados en las aulas de países como Argentina, Colombia y Ecuador. Adicionalmente se describe el proceso metodológico abordado para la revisión de la literatura bibliográfica, lo que permitió establecer algunas conclusiones acerca de los aspectos que determinan la afectividad aplicada o no en los salones de clases.

\section{Perspectiva teórica}

\section{Afectividad}

Entendemos la afectividad como el vínculo que se establece entre personas que se relacionan, generando una interdependencia de influencia mutua; se puede afirmar, entonces, con Chaves (1995) citado en González, M. \& González, A.(2000) manifiesta que la afectividad es un conjunto de sentimientos expresados a través de acciones entre personas en cualquier contexto social en que estén inmersos los individuos.

En este mismo sentido Maiorana (2010), expresa que los espacios educativos, incluidos los de formación académico-profesional son también contextos de desarrollo afectivo. Si la vida emocional es la base de la felicidad humana, una buena relación afectiva será el ingrediente fundamental para el aprendizaje. No se aprende sin el vínculo, incluso en los espacios donde prima lo cognitivo.

Por otro lado, Valencia, Páez y Echeverría (1989) y Fiske \& Taylor, (1991), empíricamente han encontrado que los estados de ánimo positivos facilitan el aprendizaje y la ejecución (aumentan el auto-refuerzo y las respuestas altruistas y de generosidad, influyen positivamente la percepción y 
el recuerdo, aumentan la sociabilidad y el contacto social.

Martínez (2009) sostiene: "El sistema educativo predetermina las funciones que, tanto el docente como el estudiante, deberán asumir en los contactos interpersonales que se producen en el medio escolar" (p.60). Esto se traduce en que saber convivir logra que los seres humanos se desenvuelvan de manera armoniosa en los distintos espacios en que se involucran. Los docentes, en su accionar cotidiano tienen que esmerarse en el manejo del entorno social; compartir sentimientos, experiencias del medio, actitudes y valores beneficiosos en la formación personal y social de cada individuo.

De acuerdo con lo anterior, Herbart (1806) en Touriñán López (2013), propone la doctrina de la instrucción moral como base del conocimiento, con el objetivo de convertir al individuo en un ser virtuoso. El imponer reglas a los niños, acompañado de la amabilidad y la tolerancia, coadyuva a la retroalimentación oportuna, aspecto fundamental que sirve como motor de toda actividad pedagógica.

García Molina (2013) manifiesta que el profesor que ostenta un saber obtenido por investigación y que se autoriza a transmitirlo parece haberle alcanzado la fecha de caducidad. El signo de los tiempos empuja a trabajar en una suerte de desierto cultural tomado por la doble exigencia de la aplicabilidad inmediata de los contenidos y de una actitud cercana, comprensiva y altamente emotiva.

En virtud de esto Macherey (2011) expresa que la figura del profesor práctico, del profesor tutor, del guía, del amigo comprensivo y disponible debe hacer agonizar el saber transmitido desde la tarima, y de su sustento último: la autoridad epistemológica del docente. El profesor se convierte en consejero, en tutor, en benevolente hermano grande resignado a un laisser-faire para orientar mejor al estudiante.

De acuerdo con Escámez, (2010), el procedimiento que suelen usar los docentes es el siguiente: formulan una pregunta o problema intrigante $y$, siempre que sea posible, conectado con la realidad cotidiana. Ayudan a los estudiantes a comprender con claridad la pregunta o problema; los comprometen a actividades intelectuales superiores, solicitan respuestas argumentadas a las preguntas. Así consiguen la atención de los alumnos, puesto que focalizan la asignatura en ellos y en los problemas que les preocupan, buscan que se comprometan en las respuestas, provocan su trabajo fuera del aula y atraen a los escolares al razonamiento desde la disciplina. La excelencia del profesor también requiere buena oratoria expositiva, un lenguaje cálido con los alumnos, dar las explicaciones que se le demandan y escuchar atentamente a sus estudiantes.

\section{Consecuencias del desinterés afectivo}

En Chile, Arón y Milicic (2000) identificaron elementos que inhiben el desarrollo socioemocional de los estudiantes y, a su vez, favorecen la violencia escolar. Esta información derivada fundamentalmente de sus estudios cualitativos, estos fueron: una concepción autoritaria de la educación, una rigidez excesiva en el concepto de jerarquía, sistemas de control coercitivos, una creación unidireccional de las relaciones de respeto (tú me respetas a mí), una relación de la obediencia que no permite la divergencia ("porque sí"), y la evasión del conflicto. Este pronóstico permite visualizar consecuencias negativas para la educación latinoamericana, no solo porque se lo haya palpado en un centro educativo desde muy temprano, sino porque se lo detecta, pero no se hace algún plan o programa encaminado a resolver estos conflictos internos como la aparición de un ambiente de indisciplina, desorganización y escaso interés del estudiante al trabajo escolar, un bajo rendimiento académico y, una evitable deserción escolar, todo esto, debido a la poca autoestima que posee de sí mismo el estudiantado que no es querido.. Mena y Vizcarra (2001) plantearon que estos criterios definirían a los sistemas escolares maltratadores y maltratados, las que desembocarían, más temprano que tarde, en un abismo sin final.

Dicho esto, Brito (2010) enfatiza que desde hace varias décadas, las formas y sentidos históricos de la escuela secundaria dan prueba de su baja eficacia para resolver los problemas relativos al fracaso escolar y la exclusión de los alumnos ya que tradicionalmente, no se ha dado la suficiente relevancia a los factores afectivos involucrados en la práctica educativa en las aulas. Sin embargo, si realizamos un ejercicio 
personal de recuerdo de nuestros años escolares, muy probablemente constatemos que algunos de los episodios que con mayor nitidez vienen a nuestra memoria y pueblan nuestras narrativas infantiles son eventos fuertemente entramados con experiencias emocionales intensas (positivas o negativas). En ese mismo sentido Valdés (200), sostiene que siempre quedan aquellos sentimientos y afectos que dibujan los contornos de aquella inolvidable maestra de los grados inferiores, disgustos ante alguna tarea escolar, risas compartidas con algún compañero de clase y la felicidad o frustración frente al resultado de una evaluación.

Estas vivencias inducen a Saint-Onge (1997) a manifestar que los centros escolares están llamados a organizar y dirigir el proceso formativo al alcance del desarrollo de los ciudadanos hacia las demandas sociales actuales. Dar clases es algo más que ofrecer una disertación oral, es aplicar un método de enseñanza que ayude eficazmente a que los alumnos se pongan a aprender con interés, método ideado y planificado por los que enseñan En palabras de Ortega y Gasset (1930), ciertamente, cuando una nación es grande, es buena también su escuela. No hay nación grande si su escuela no es buena. Pero lo mismo debe decirse de su religión, de su política, de su economía y de mil cosas más. La fortaleza de una nación se produce íntegramente. Si un pueblo es políticamente vil, es vano esperar nada de la escuela más perfecta.

\section{Criterios que caracterizan la afectividad en el ámbito escolar}

En la revisión teórica y documental se muestra que el enfoque predominante hace énfasis en los procesos que facilitaron la afectividad y sobre los efectos positivos del mismo. A pesar de su importancia, parece no haberse explorado con más detenimiento, aunque está considerado como un aspecto primordial dentro de los objetivos de la educación (Solís Ramírez, E., Porlán Ariza, R., Rivero García, A. y Martín Del Pozo R., 2012).

En este sentido, Touriñán (2013), muestra tres vertientes de la afectividad desde el punto de vista psicológico y pedagógico, esta son preponderantes en el momento de aplicarla dentro del salón de clases. Los criterios fueron seleccionados con base a esos interrogantes sobre la calidez y la calidad de educación que se plantean los autores. Por lo pronto se ha abordado los siguientes: la motivación, la inteligencia interpersonal que maneje el profesor y la sensibilidad con que el educador se ubica frente a sus discípulos.

\section{Motivación}

De acuerdo con Baracho (2010), en psicología y filosofía, motivación se define como los estímulos que mueven a la persona a realizar determinadas acciones y persistir en ellas para su culminación, es decir, es emulsionar a alguien a iniciar acciones encaminadas a conseguir objetivos. En cuanto al aprendizaje, se dice que es un proceso que puede ser conceptuado de forma sintética, es el modo con que las personas adquieren nuevos conocimientos, desarrollando competencias y cambiando comportamientos.

Como lo indican Gilbert (2005) y Bono (2010), uno de los aspectos que aprendieron en sus años de formación como profesor, es que la enseñanza tiene que ver con las relaciones, en virtud de esto, la motivación para aprender solo puede ser comprendida a partir de su estudio en la clase, entendida como un contexto específico de aprendizaje. De manera que es importante estudiar cómo es que los alumnos pueden ser motivados en diferentes direcciones a partir de la influencia del desempeño docente. Particularmente, la relación que se establece entre alumnos y profesores puede ser el aspecto más relevante a tener en cuenta para conocer la motivación en general y más específicamente el interés por aprender. Dentro de esta concepción McLean, (2003), afirma que el manejo del feedback es posiblemente la guía más importante para obtener información sobre la incidencia motivacional que los profesores producen en los estudiantes.

\section{Inteligencia interpersonal}

Brites de Vila, G. y Almoño de Jenichen, L. (2002), definen la inteligencia interpersonal, como la habilidad para establecer relaciones con otras personas e interactuar con ellas. La sensibilidad especial para comprender sentimientos, pensamientos e interpretar la conducta de los demás, captar estados de ánimo, sentir lo que otros sienten poniéndose en su lugar, la flexibilidad para entender otros puntos de vista, la capacidad para asumir diversos roles dentro de un 
grupo. La aptitud para llegar a los demás, asesorar, persuadir, liderar, negociar, actuar como mediador. Es la curiosidad e interés por distintos estilos de vida. Es comunicarse en forma efectiva, expresándose con claridad e interpretando adecuadamente los mensajes.

Según González (2011), los estudios de inteligencia múltiples desarrollados por Howard Gardner, hacen referencia que la inteligencia interpersonal se define como la capacidad de comprender a los demás seres humanos, pudiendo lograr una mejor interacción y empatía. Esta nos ayuda a comprender a los demás, poder contextualizar sus comportamientos, estados de ánimo y deseos. Si logramos comprender lo que el otro necesita y en qué momento lo necesita, alcanzaremos una mejor relación humana con el entorno a través de la óptima capacidad de comunicación.

De acuerdo con Carrasco (2014), la construcción de significados compartidos entre profesor y alumno está íntimamente ligada a la construcción de un vínculo afectivo interpersonal en el que se ponen en juego no solo los sentimientos, las motivaciones y las expectativas de los alumnos, sino también las del profesor. La experiencia pedagógica, el enseñar y el aprender, se desarrollan en el vínculo: tienen una dimensión histórica, intersubjetiva e intrasubjetiva.

\section{La sensibilidad en el afecto}

Hablar de sensibilidad implica tacto, una percepción consciente y estética, un agudo sentido de qué hacer o decir, para mantener las buenas relaciones con los demás o para evitar la ofensa (RAE, 2014). Hay que reconocer que tiene propiedades interpersonales y normativas que parecen especialmente adecuadas para las interacciones pedagógicas con los niños. El tacto pedagógico es una expresión de la responsabilidad que se asume al proteger, educar y ayudar a los niños en su desarrollado. Es así que se manifiesta principalmente, como una orientación consciente en cuanto a la forma de ser y actuar con los niños. No se trata tanto de la manifestación de algunos comportamientos observables como de una posición activa en las relaciones. Aun así, existen varias maneras de describir cómo se manifiesta en la forma pedagógica de la atención de alumnos con necesidades educativas o sin ellas (Zúñiga, 2010).
Desde el supuesto pedagógico del que partimos, Omart y Brunetti (2009) manifiestan que no se puede aprender ética sin la posibilidad de vivir una experiencia que nos lleve a la reflexión. De ahí que postulemos la necesidad de una educación de la sensibilidad moral no solo en el niño sino también en el adulto. ¿Cómo podemos transformar a este individuo pasivo a que se convierta en una estudiante sensible y poder desarrollar la afectividad? Por parte de los docentes, se debe diseñar, ejecutar $\mathrm{y}$ evaluar experiencias vivenciales que involucren la sensibilidad de los alumnos y por parte de los estudiantes, vivir experiencias que permitan al alumno ponerse en el lugar del otro.

Poro otro lado, Acosta, Arias, Ortega, Quintero y Gómez Sánchez (2003) aciertan a decir que la afectividad permite que nos relacionemos con el mundo, consistiendo en la capacidad y la experiencia cambiante de ser afectado por el entorno. Mientras que Sánchez (s.f.) afirma que hablar de afectividad es hablar de una capacidad propiamente humana y una necesidad que, dependiendo de la forma en que se desarrolle, orienta el comportamiento de la persona frente a sí misma y frente a los otros, en consecuencia de ello, al ser la afectividad un tema tan importante en la vida de las personas y la sociedad, se encuentra la necesidad de indagar en este dentro de la institución, ya que se presentan interacciones sociales que generan sentimientos y vivencias desagradables y dolorosas para niños, docentes y funcionarios, lo cual, interfiere en la realización y el desarrollo personal y comunitario.

En definitiva, la actitud del docente ante su profesión y su ejercicio, que lo lleva al éxito de los resultados esperados frente a los grandes cambios y avances del sistema educativo, debe estar enriquecida de conocimientos y estrategias que le permitan asegurar logros significativos en su quehacer pedagógico, que comprende un proceso de construcción de conocimientos en los estudiantes y no la sola adquisición de respuestas. Las personas aprenden y entienden mediante la interacción con su entorno, y con estas estrategias que aprendió, las utiliza para desarrollar habilidades y conocimientos (Piaget, 1932). 


\section{Metodología}

Este trabajo investigativo es una reflexión en torno a la relevancia del factor afectivo dentro de las salas escolares como motivador e impulsador de unas excelentes relaciones interpersonales en el ámbito educativo. En este sentido, se empleó una metodología con enfoque cualitativo, de tipo descriptiva acerca del grado de afectividad en la cátedra de Argentina, Colombia y Ecuador, utilizando un muestreo por conveniencia, debido a que los países analizados, tienen información disponible en internet en relación a la afectividad en el aula y los criterios sobre el tema expuestos por los diferentes investigadores de los países analizados, considerando tres criterios de análisis acerca de la afectividad: la motivación por parte del profesor, la inteligencia interpersonal del maestro y la sensibilidad del educador.

El análisis se llevó a cabo mediante la revisión bibliográfica de literatura suramericana, cuyas ponencias explican el valor de la afectividad como un requisito primordial en el proceso enseñanza-aprendizaje, exponiendo sus conclusiones sobre cómo se aplica el uso de la afectividad en el aula.

El proceso metodológico que se siguió fue definir el concepto de afectividad y las vertientes que la caracterizan. Analizar y extraer las ideas esenciales, bajo los criterios mencionados que comparten los investigadores de los países referidos, y, por último, sintetizar a través de un cuadro comparativo, cómo se hace efectivo el uso de la afectividad dentro de las aulas estudiantiles.

En el presente trabajo no se aplicó la metodología en un universo más amplio debido a la falta de información actualizada en otros países, lo que hubiese generado mayores datos para obtener una visión más amplia de este problema abordado por otros pedagogos, educadores y psicólogos, en diferentes épocas.

Esto conlleva finalmente a presentar los resultados estudiados, permitiendo exponer en síntesis los países en donde se está gestionando de manera eficiente la afectividad y la motivación por parte del profesor, la inteligencia interpersonal del maestro y la sensibilidad del educador dentro del salón de clase.

\section{Resultados}

Los resultados que se presentan a continuación son producto del análisis e interpretación de la revisión bibliográfica de los pensamientos o postulados de varios investigadores de Argentina, Colombia y Ecuador, lo que hizo posible establecer aspectos comparativos entre estas naciones.

\section{Caso Argentina}

\section{Motivacion}

En lo que respecta a Argentina Brito (2008), manifiesta que la motivación constituye una dimensión que cobra particular fuerza en la definición de la experiencia que los docentes construyen en el cotidiano escolar. Pero se trata de una dimensión que no solo representa una demanda para los profesores en relación con sus estudiantes sino que también los alcanza a ellos. La siguiente reflexión de una profesora entrevistada condensa la necesidad de la propia motivación para asumir la tarea pedagógica: «A veces digo, ¿y si me pongo yo delante de la clase para hacer lo mismo que ellos tienen que hacer para ver si los motivo?». En este aspecto hay docentes que buscan formas para captar la atención y el interés de los alumnos, y de este modo encontrar reconocimiento, implica la búsqueda de la propia motivación .

Sin embargo, lo que también vale remarcar es que los docentes especialmente las mujeres, manifiestan encontrar satisfacción en el intercambio, diálogo e interacción con sus alumnos. La identificación positiva de esta cuestión se complementa con la alta valoración que hacen sobre su vínculo con los alumnos que, con solo algunas diferencias positivas por parte de aquellos que se desempeñan en escuelas privadas, es calificada entre «buena» $(86,2 \%)$ y «regular» $(13,8 \%)$, pero en ningún caso como «mala» (Brito, 2008).

Para Dussel, Brito y Núñez, (2007), la opinión de los profesores sobre las razones del abandono escolar en la escuela secundaria es que el $27,3 \%$ de los estudiantes desertan como consecuencia de la falta de motivación por parte de los educadores: el aburrimiento en la escuela. 
De acuerdo con López, V., Bilbao, M., Rodríguez, J. I. (2012) existen diferentes climas dentro de las aulas escolares, cuya influencia en la calidad de las relaciones y los aprendizajes es preponderante porque genera contextos de tolerancia y de motivación académica $\mathrm{o}$, en su defecto, originan caos y temor en el escenario escolar.

\section{Inteligencia interpersonal}

Por otro lado, para poder acercarse a otros y comenzar una conversación hay que ser capaz de leer ciertas claves contextuales (por ejemplo, si la otra persona no está ocupada o dialogando con otros). Con frecuencia Mario (Nombre cambiado para proteger identidad), se siente rechazado porque no puede ser capaz de comprender esas claves y generar estrategias para acercarse a sus pares. Además, si siempre que se acerca es para hablarles solo de lo que a él le interesa, los demás tienden a alejarse. Como tiene un alto nivel de "inteligencia interpersonal" tiene consciencia de que se queda solo y manifiesta que no consigue amigos. Esto lo pone muy triste. Necesita ayuda para crear "puentes" hacia los demás. No puede hallar las claves necesarias, en cada situación interpersonal, para tener éxito en establecer vínculos. Y este es un punto importante en el problema del síndrome. No es que a Mario no le interesen las personas. Pero personas y relaciones humanas en general son una especie de "misterio" para él. Así como para los demás pueden constituir un "misterio" la forma de ser de Mario.

Los docentes, están llamados a promover encuentros y gestiones de convivencia, dentro del contexto escolar donde se busca afianzar los logros de los estudiantes a través de las relaciones interpersonales para fortalecer así el desarrollo de actitudes positivas que permitan la interacción del maestro con el educando en un clima de confianza y de cooperación: "Cuando alguien, con la autoridad de un maestro, describe el mundo y tú no estás en él, hay un momento de desequilibrio psíquico, como si te miraras en el espejo y no vieras nada" (Adrienne Rich, 2004:1).

La educación se basa principalmente en las relaciones interpersonales y la experiencia docente indica que los procesos de enseñanza-aprendizaje no dependen solo del conocimiento y de la capacidad intelectual de quienes participan, sino también de su saber emocional. El saber emocional es una competencia imprescindible en el profesorado, sobre todo si partimos de que constituye un modelo de aprendizaje socio-emocional de gran impacto para los alumnos, y además, porque el manejo de la afectividad, supone, ante todo, el conocimiento de nosotros mismos y del otro (Maiorana,2010).

"El profesor que se muestra honesto, empático y competente, que reacciona ante las adversidades y los temas desconocidos con sinceridad y que valora a los aprendices y se les acerca para aprender juntos, despierta comportamientos sanos, curiosidad y motiva creando un clima propicio para el aprendizaje. Lo dicho no implica negligencia dejando pasar errores, sino optar por la retroalimentación permanente y enriquecedora" (Rodríguez, 2011:4).

\section{La sensibilidad en el afecto}

En Argentina se plantean proyectos cuyos objetivos ponen en marcha procesos dinámicos para el desarrollo social, promoviendo la convivencia pacífica, en donde los docentes se sensibilicen frente a los problemas que suelen presentar los estudiantes que están bajo la tutela del profesorado. Entre las actividades realizadas está el llamado "Taller de sensibilización", se explica en grandes rasgos el modelo, de esta manera los docentes, sensibilizados, incorporan la temática a su planificación curricular.

El rol que juegan los maestros y directores de las escuelas es importante en el momento de presentarse alguna dificultad, puesto que los docentes, en muchas ocasiones, asumen el papel de coordinadores, apoyando a los estudiantes en la mediación y en las trabas que esta pueda presentar, convirtiéndose en sustento para los estudiantes. Desde esta experiencia piloto en 1997 a la fecha (2008), el modelo se ha instalado en 21 escuelas de la ciudad de San Carlos de Bariloche, beneficiando a más de 10.000 estudiantes y ha logrado la disminución de los hechos violentos entre los alumnos, así como una relación más armónica entre estos y los docentes. Los postulados de estos objetivos son: Enseñar a los niños, niñas y jóvenes, actos pacíficos para afrontar sus conflictos y borrar la violencia como solución (Fundación Alternativa, Social y Educativa, 2008). 


\section{Caso Colombia}

\section{Motivacion}

El colegio Champagnat ubicado en la Localidad de Teusaquillo en la ciudad de Bogotá, es un centro educativo Marista, en una única jornada de carácter privado, que destaca en lo académico por poseer alta tecnología, asociación con varias universidades y la implementación de escuelas de formación. Además este colegio es pionero y modelo de Educación en la Ecología y el Medio Ambiente.

Por tal motivo, fue escogido para llevar a cabo una investigación con un enfoque cuantitativo en el que participaron 33 estudiantes entre los 16 y 17 años de edad, todos de género masculino, dentro de un estrato social medio y medio alto. la muestra pertenece a estudiantes de la sección $\mathrm{C}$ del grado undécimo, cuya selección obedece a que todos pertenecen al mismo nivel avanzado de conocimiento de lengua extranjera. La recolección de datos se basó en la aplicación de métodos con medición numérica y abordó la problemática de cómo crear actividades didácticas de motivación y que sean acogidas dentro del grupo de adolescentes, que a su vez sean efectivas para el aprendizaje de los mismos.

Dentro de esta investigación se tiene en cuenta la fundamentación teórica de los autores como es el caso de Morrison (2005) quien toma como referencia la teoría de Maslow (1998) Hombre autorrealizado, hacia una psicología del ser, donde la motivación es también llamada la auto-realización que se basa en la satisfacción de las necesidades humanas. De este modo Maslow identificó la auto-realización, como la mayor de las necesidades del ser humano. Sin embargo, se afirma que los niños y los adultos consiguen la auto-actualización cuando las necesidades básicas están satisfechas, haciendo énfasis en que la motivación es importante en el aprendizaje del ser humano, ya que cuando este se encuentra motivado logra involucrase en actividades que lleven a niveles superiores del aprendizaje.

En un año escolar de seguimiento a los estudiantes del grado undécimo del Colegio Champagnat de Bogotá, se pudo evidenciar los logros de los estudiantes al obtener una motivación acorde con los procesos de enseñanza impartidos por la docente de lengua extranjera, creando en ellos, una verdadera competencia comunicativa, la investigadora afirmó que en Colombia es importante mejorar las actividades dentro del aula para así lograr en nuestros estudiantes afianzamiento de las habilidades comunicativas (Nielsen, J. 2010).

\section{Inteligencia interpersonal}

Dentro del mundo de la escuela, tal vez es el aula de clases donde se ponen en escena las más fieles $\mathrm{y}$ verdaderas interacciones entre los protagonistas de la educación intencional, maestros y estudiantes. Una vez cerradas las puertas del aula, se da comienzo a interacciones de las que solo pueden dar cuenta sus actores. Es aquí donde el maestro se hace y se muestra, aquí ya los deseos se convierten en una realidad, ya no es el mundo de lo que podría ser, sino el espacio de lo que es.

En Colombia, se trata de buscar soluciones a los momentos de crisis que viven los colombianos. El maestro, en palabras de May (2001), debe ser el agente que enseñe, investigue, trabaje, cree una pedagogía del afecto, que haga descubrir cada individuo, su potencialidad y se eduque con las experiencias compartidas entre ellos. Todo debe moverse en torno al alumno. Esta interrelación debe convertirse en un vínculo que lo reconozca como persona.

El educador tiene en sus manos sujetos reales a quien debe respetar como persona Tal como se dijo, el educador trabaja, con individuos que sienten, desean y sueñan, es decir, un ente que es persona con todas sus facultades para disentir y discernir, lo que significa que la pedagogía dentro del aula debe girar en torno al alumno y dejar de moverse alrededor del docente (May, 2001).

\section{Sensibilidad en el afecto}

En la Institución Educativa Roberto Cavelier, colegio distrital y localizado en la zona Aguanica en Cajicá, Colombia, se efectuó una investigación con una aproximación etnográfica durante un año, compartiendo con la comunidad y construyendo con esta las representaciones sociales que ellos tienen de la afectividad. 
En el trabajo con niños, niñas y profesores de la institución, se encontró un marcado interés por el tema de afectividad, que se considera como elemento fundamental dentro del desarrollo integral del ser humano, entendiéndolo como el hecho de brindar afecto al otro. Sin embargo, en las relaciones interpersonales cotidianas se presentan actos contradictorios con tales concepciones.

Se pudo constatar que los miembros de la comunidad manifestaban necesidades acerca de sus vivencias acerca del trato, el respeto, la forma de comunicarse, los golpes, gritos y la forma de reconocer al otro. Por ello, la investigación se basó en las representaciones sociales acerca de la afectividad, con el fin de conocer y construir con la comunidad tales representaciones y su reflejo en la vida cotidiana, de forma que fuera más deseable para ellos.

\section{Caso Ecuador}

\section{Motivación}

En lo que respecta a la República del Ecuador, en el plan decenal(2006-2015), elaborado por el ministerio de Educación, se manifiesta como objetivo prioritario brindar calidad y calidez de la educación, cuyos ambientes de aprendizaje evidencien el buen trato y el sentido humano del docente hacia el alumnado, sin embargo las autoridades dan la posibilidad a que el investigador aplique este proyecto en las instituciones que creyere conveniente (Peláez,2012).

En virtud de esto, se llevaron a cabo dos estudios sobre la motivación y su influencia en el proceso de enseñanza-aprendizaje en el nivel inicial y en el nivel medio respectivamente. En lo que respecta a la investigación de Educación Inical, esta estuvo comprendida en el periodo 2010-2011, las maestras de las cuatro escuelas que estuvieron inmersas en ese trabajo investigativo, pertenecientes a la ciudad de Machala (Ecuador), manifestaron que el aspecto motivacional es fundamental en la atención a los niños cuya edad comprendía entre los 3 y 4 años, por lo que siempre el ambiente y el espacio del aula deben mostrar una armonía y aprecio constante para lograr la adaptación al entorno educativo. La motivación es un factor necesario en todo momento y lugar, con la finalidad de llamar la atención o impulsar un agradable ambiente antes y durante la intervención que realice toda persona; en la expresión, y en la forma de actuar, se lo debe hacer con motivación lo cual los gestos en especial son fundamentales para lograr llamar la atención en este caso de los niños y niñas (Valverde, 2011).

La segunda investigación de campo fue comprendida en el periodo (2012-2013), el instrumento evaluativo fueron los cuestionarios CES de Moos y Trickett, elaborado de acuerdo a la realidad educativa ecuatoriana, realizada en dos centros educativos ubicados en el sector Urbano del Cantón Gualaquiza, la escuela Pedro de Vergara con una muestra de cuatrocientos veinte estudiantes de cuarto y sépimo año con mujeres y hombres y el colegio Fiscomisional Río Cenepa con un total de 650 estudiantes. Los resultados obtenidos reflejaron que los docentes se preocupan por crear buenos ambientes y que todos sus estudiantes se integren en el momento de la clase, mientras que los estudiantes demostraron ser participativos, ordenados, obedientes a las reglas y motivados dentro del salón, a pesar de que hubo un porcentaje ínfimo de alumnos que reflejan una actitud de desinterés (Peláez, 2012).

\section{Inteligencia interpersonal}

La Unidad Educativa Mario Rizzini de la ciudad de Cuenca, Ecuador, en el periodo 2010-2011, efectuó una investigación cuya hipótesis radicaba en que la inteligencia emocional incide de manera directa en el desempeño docente, por ende, se refleja en las relaciones con el estudiantado. Este importante factor se encuentra en el discurso institucional y se lo trabaja en ciertas áreas de acción, pero no se localiza como macro objetivo en su desarrollo fundamental. De esto se deriva una propuesta puntual de capacitación que favorezca las dimensiones psicológica, pastoral, desarrollo personal y educativo-cultural. Al asumir este nuevo enfoque de aplicación de la inteligencia emocional se considera que se ganaría por partida doble, mediación y ayuda a los estudiantes en requerimientos muy puntuales y solución de conflictos y mejoramiento del desempeño docente, lo que se traduciría como equilibrio personal y madurez profesional. Por supuesto, que esto implica manejar un ideal de perfil docente, que genere un compromiso hacia la mejora continua y una actitud transparente para aplicar procesos de innovación en el aula, que se 
concretan en resultados de bienestar y un entorno de aprendizaje dinámico, activo e inclusivo.

Es así que dentro de sus conclusiones de investigación indican que la inteligencia emocional influye de manera determinante en el sujeto, y no solo en su parte emotiva, sino también física, sin importar el entorno del que se trate, personal, familiar o social. Las actitudes y comportamientos siempre van en correspondencia directa con los aspectos de formación del sujeto y a medida que alcance su madurez entonces la persona se mostrará mucho más equilibrada, consistente y coherente.

Las emociones tienen vinculación directa con el aprendizaje, un proceso que se construye por los actores de manera colectiva y sobre la base de la mediación pedagógica, término bastante desarrollado por Lorenzo Tébar en su libro El profesor mediador del aprendizaje (2009) cuya intencionalidad es educar para la autonomía y la libertad. Para la Unidad Educativa Mario Rizzini, desde una perspectiva salesiana, se traduce como acompañamiento, animación de procesos y propuestas para el desarrollo integral del sujeto.

\section{Sensibilidad en el afecto}

El mejor entorno para aprender es un ambiente de cordialidad, afecto, respeto y de buenas relaciones interpersonales entre autoridades, docentes, alumnos y padres de familia. Autoridades comprometidas con el progreso de la institución; docentes motivados, satisfechos y orgullosos del trabajo que desempeñan; estudiantes alegres, libres de situaciones de violencia o conflictos con sus compañeros o con sus maestros; padres de familia que apoyan, valoran, que brindan cariño y comprensión a sus hijos; creando de este modo un clima escolar y de aula que sigue el camino de la eficacia y calidad educativa.

En la Provincia del Azuay el investigador Rafael Peralta, realizó una investigación de campo a los estudiantes del séptimo año de educación básica de la escuela fiscal mixta urbana "República de Colombia" y de la escuela fiscal mixta rural "Juan Bautista Vázquez". Los criterios de selección se debió a la poca tecnología con que cuentan estos centros y permiten diferenciar las relaciones interpersonales entre una escuela mixta rural de la urbana. Por ese motivo, el cuestionario aplicado fue el de clima social escolar CES de Moos y Trickkett. Este instrumento de evaluación posee 134 ítems que igualmente hacen referencia a cuatro dimensiones, la dimensión considerada fue el clima social del aula, es decir, observar las relaciones alumno-profesor y profesor-alumno desde la percepción del estudiante, lo que permitió realizar un análisis cuantitativo y luego cualitativo.

Los resultados obtenidos por Peralta (2012), muestra que los alumnos del plantel rural Juan Bautista Vázquez, sobresalen gracias al buen trabajo de la docentes y se sienten respetados, además que los conflictos del aula se solucionan de manera profesional, no obstante el 59\% de estudiantes afirma que nunca se da solución a estos problemas de una manera pacífica y paciente por parte del profesor, lo que origina la falta de espacios alternativos para mejorar la comunicación y el trabajo con los educandos, ocasionando la pérdida de oportunidades para corregir falencias que no han sido identificadas en los espacios normales de clase.

En lo que respecta la segunda aplicación del cuestionario CES a los estudiantes del séptimo año de Educación Básica de la Escuela urbana "República de Colombia", Peralta (2012) resalta que la profesora da estímulos a los estudiantes cuando realizan un buen trabajo, valora los trabajos grupales de los estudiantes y les da una calificación; sin embargo, las opiniones de ellos no siempre son consideradas ni escuchadas por la docente, lo que incide en un desconocimiento por no saber la percepción que tiene el estudiante en cuanto al aprendizaje que está recibiendo.

\section{Discusión de resultados}

En los casos de los países analizados, se puede afirmar que los ambientes hostiles causan un deterioro en el desempeño estudiantil, pues la carencia de afectividad crea vínculo personal poco satisfactorio en todo el engranaje educativo. Existe un contraste entre la teoría y la práctica, mientras que los postulados teóricos recomiendan una constante aplicabilidad de afecto en los salones de clase, esta situación no es real, debido a que en la práctica aún no se aborda de manera satisfactoria las propuestas enfatizadas por los diferentes investigadores, cuya importancia influyen positivamente en el contexto diario estudiantil. 
Para los investigadores de la República de Argentina y Colombia, hay una marcada preocupación para la docencia argentina y colombiana en hallar estrategias idóneas y oportunas como solución en el resquebrajamiento de las relaciones interpersonales en este encuentro diario, ante esta circunstancia, la sensibilidad que todo maestro muestre a sus tutelados, el escucharlos y el mediar conflictos, se constituyen en muestras de interés hacia ellos, y por ende está escuchando los problemas de la comunidad educativa. $\mathrm{Su}$ trabajo dentro de un clima de respeto y de escucha activa es vital en el aumento de la autoestima del estudiantado, lo que produce el mejoramiento del rendimiento escolar.

No obstante, para Ecuador, solo pocos investigadores se pronuncian sobre este concepto como principio fundamental del proceso educativo. La comunidad educativa ecuatoriana posee muchas ganas por entender a su alumnado y convertirse en mostrar modelos de enseñanza, sin embargo, no existe un pensamiento unificado para la elaboración de proyectos con estrategias pedagógicas que coadyuven al acercamiento entre docentes y los adolescentes o jóvenes con la finalidad de mejorar el rendimiento académico y constituir al educando ecuatoriano en un individuo con mejor autoestima.

En cuanto al criterio de la inteligencia interpersonal, Colombia y Argentina, consideran que este es un factor vital para el desenvolvimiento de un correcto clima de aula, en donde se pueden intercambiar roles, compartir ideas y relacionarse de una manera cercana sin perder el respeto y la privacidad de cada uno de los involucrados en este sistema. En cambio, los investigadores de la República del Ecuador consideran que este aspecto es importante, pero no es esencial dentro del contexto educativo.

Además, haciendo referencia a la motivación como factor impulsador de la afectividad, los investigadores argentinos y colombianos, afirman que esta característica es lo que constituye un pilar preponderante como alentador de un buen clima escolar y de un mejor entendimiento entre maestros y alumnos para la consecución de las metas propuestas al inicio de toda clase, mientras que existen pocos investigadores ecuatorianos que manifiestan la relevancia de este aspecto en toda circunstancia, por otro lado, se debe realizar una investigación más exhaustiva sobre este fenómeno que nos atañe a todos.

Se advierte también, que la preparación pedagógica del maestro pone en consideración el conocimiento de los diferentes estilos de aprendizaje para comprender cómo se enseña a las diferentes individualidades. Bajo esta perspectiva, se pone de manifiesto que en determinadas situaciones se vislumbra la necesidad de desarrollar estrategias pedagógicas modernas e innovadores, ya que no hay un material didáctico y empleo de tareas grupales que impulsen la libre asociación, sin motivar ni sensibilizar al alumno dentro del aula, incluso en determinados centros escolares, los educandos no son escuchados, menos aún sus opiniones son consideradas. Este aspecto no enriquece la labor docente, puesto que el maestro que no está plenamente identificado con su grupo de clase, no podría dar muestras de sensibilidad, ser afectuoso o motivar a su estudiantado, al éxito.

En definitiva, en los planteles de los países en donde se aplicaron encuestas u observaciones sobre el comportamiento y estrategias afectivas, se refleja a un estudiantado que dista mucho de comprender que él es un individuo con facultades valiosas y con potencialidades que lo constituyen en un ente con derechos y con plena voluntad para tomar decisiones que lo ayuden a ser mejor persona. En consecuencia de ello, se hace necesario analizar que las actitudes de padres de familia, maestros y autoridades dejan una huella decisiva en la personalidad de este niño $o$ adolescente que se siente seguro en el momento de su escolaridad o nervioso y dubitativo, si en la misma familia el trato ha sido poco amable.

\section{Conclusiones}

Una vez realizada la revisión bibliográfica, se concluye que existe la necesidad imperante de establecer entornos favorables dentro de las aulas de clase, donde los estudiantes alcancen altos niveles de interés afectivo que los ayuden a un mejor desenvolvimiento en el área académica, los centros educativos no son espacios solo para lo cognitivo o el saber intelectual, sino también para profundizar de mejor manera los estados emocionales y encuentros vivenciales de cada sujeto en el ámbito escolar. 
En este sentido, el análisis de la literatura sobre la concepción teórica de la afectividad por varios autores, fue pertinente porque se pudo constatar que el trato amable dentro del salón de clases es un factor protagónico para el desempeño escolar, enfocando la importancia de que los procesos cognitivos van correlacionados con los afectivos, debido a que ambos factores conducen a un desarrollo integral del educando.

En el proceso metodológico se pudo visualizar que hay una preocupación sobre el tema de la afectividad del maestro hacia el alumnado. Se considera, entonces que el factor decisivo para mantener unas excelentes relaciones personales, un mejor aprendizaje y un poder de decisión en la resolución de problemas, se debe a la motivación, la inteligencia interpersonal y la sensibilidad que lleva intrínseca el ser afectivo. Dicho esto, si el ambiente es tenso y poco dinámico en el aula escolar surgirá un rendimiento académico poco satisfactorio.

Por otro lado, se evidencia que los docentes que mantienen una expresión diáfana, cordial y de sonrisa franca, tienen más ventajas que aquellos que muestran seriedad ante sus estudiantes, quienes tienden a percibir distanciamiento $u$ hostilidad. Sin embargo, la sonrisa no es sinónimo de franqueza, ya que puede ocultar lo que verdaderamente ocurre en el interior de cualquier ser humano que finja una postura falsa. Lo que sí es cierto es que los docentes, que tratan amablemente a sus dirigidos, tienen la ventaja de ser escuchados con más facilidad.

En definitiva, la afectividad es un proceso que va desde lo sentimental y emocional hasta lo racional: pasando por lo afectivo hasta lo intelectual y es recíproco, porque comienza de los maestros al alumno y viceversa, generando un mejor rendimiento escolar y construyendo una mejor persona.

Ante esto, es lamentable constatar que los tratados teóricos de los diferentes exponentes acerca de la aplicación efectiva de la afectividad en el aula, se cumple de manera irregular, debido a que muchas instituciones educativas lo proponen por estar conscientes de su relevancia, sin embargo, en la realidad no se cumple por fallas en su ejecución.
Es valioso indicar que para futuros trabajos, se debe analizar el grado de afectividad que mantiene el profesorado ecuatoriano en el momento de dictar la clase, dentro de las instituciones oficiales y privadas. Además, determinar las causas que inciden en las agresiones dentro del salón de clases y de qué manera el campo de la investigación de este país se vincule en proyectos para aplicar la afectividad entre los estudiantes con el propósito de desterrar los casos de hostilidad entre alumnos e inclusive estudiantes-profesores.

\section{Referencias bibliográficas}

Abramovay, M. (2005). Violencia en las escuelas, un gran desafío. Revista Electrónica Iberoamericana de educación. V.38, 53-66.

Arón, A., \& Milicic, N. (2000). Efectividad escolar: un análisis comparado. Santiago: Ediciones Pontificia Universidad Católica de Chile.

Acosta, D.J., Arias, L.F., Ortega, P., Quintero, A.M., Gómez, S.M. y Sánchez, S.A. (2003). Estatuto Ontológico de La Afectividad. (Tesis de licenciatura). Colombia: Universidad de La Sabana.

Baracho da Silva, A.(2010), La relación entre motivación y aprendizaje en ele E/LE. Recuperado de:http:// revistaliter.dominiotemporario.com/doc/LA RELACION_ANA_SHELIDA.pdf.

Bono, A (2012) Los profesores en las clases. Un estudio sobre las pautas de actuación docentes en el aula de primer año universitario desde la perspectiva motivacional Argonautas N²: $153-178$ ARG.

Brito, A (2008) Los profesores y la escuela secundaria, hoy. Notas sobre una identidad en repliegue. (Tesis de maestría). Buenos Aires: Flacso.

(2009). Acerca de un desencuentro: la mirada de los profesores sobre los alumnos de la escuela secundaria en Argentina. Revista Iberoamericana de Educación. N. ${ }^{\circ} 51,139-158$.

Brito Sarabia, S. A. (2012). Aplicación de la técnica del discurso para mejorar su autoestima en los niños de sexto año de educación básica en la escuela "Club de leones" caserío San Francisco, parroquia Constantino Fernández Cantón Ambato, noviembre 2010-marzo 2011 (Bachelor's thesis). Tomado de: http://repositorio. uta.edu.ec/handle/123456789/2724

Brites de Vila, G. y Almoño de Jenichen, L. (2002). Juegos y dinámicas para multiplicar las formas de aprender utilizando al máximo las capacidades de la mente. Inteligencias Múltiples. Buenos Aires: Ed. Bonum. 
Carrasco, B. (2004). Estrategias de aprendizaje. Para aprender más y mejor. Madrid: Ediciones Rialp.

Domínguez, M. y Sánchez, I. (2013). Reestructuración de la enseñanza superior. Postmodernidad ideológica, profesionalización liberal y mercado desregulado de la educación. Athenea Digital, 13(1), 197-215. Disponible en http://psicologiasocial.uab.es/athenea/index.php/ atheneaDigital/article/view/1032-Dominguez.

Duarte D., J; (2003). Ambientes de aprendizaje: Una aproximación conceptual. Estudios Pedagógicos, 29 (1). 97-113. Recuperado de: $\leq$ http://www.redalyc.org/articulo. oa?id=173514130007 $>$.

Dussel, I., Brito, A., \& Núñez, P. (2007). Más allá de la crisis. Visión de alumnos y profesores de la escuela secundaria argentina. Buenos Aires: Fundación Santillana.

Fiske, S. T., \& Taylor, S. E. (1991). Social cognition. New York: Mcgraw-Hill Book Company.

Fundación Alternativa Social y Educativa, Experiencia e innovación social Programa de prevención de la violencia a través de proyectos. Argentina. disponible en: http://www.falternativa.org.ar

García Molina, J. (2013) Profesar la profesión de profesor. Entre el filósofo y el agente doble publicado en Athenea Digital - 13(1), 13-27.

Gázquez, J.J., Pérez-Fuentes,M.C., Carrión, J.J. \& Santiuste, V. (2010). Estudio y análisis de conductas violentas en Educación Secundaria en España. Universitas Psychologica, 9 (2), 371-380.

Gobierno Nacional de la República del Ecuador. Plan Nacional del buen vivir. Disponible en: http://plan.senplades.gob.ec/ objetivo

González, M. \& González, A. (2000).La afectividad en el aula de clase. Revista Colombia Médica, Vol. 31(1), 55-57.

González Hueso, A (2011). Inteligencias múltiples: Gardner, Goleman y Olvera A. http://www.alaya.es/2011/12/19/ inteligencias-multiples/.

González, E. (s.f.). Educar en la afectividad, Facultad de Educación Universidad Complutense-Madrid 1. Disponible en: www.slideshare.net/valfh/educar-en- la-afectividad 17/01/2013

Jung, C. (2001). Presente y futuro. Obra Completa. Volumen 10. Madrid: Trotta.

López, V., Bilbao, M., Rodríguez, J. I. (2012). La sala de clases sí importa: incidencia del clima de aula sobre la percepción de intimidación y victimización entre escolares. Universitas Psychologica, 11(1), 91-101.

Macherey, Pierre (2011). La parole universitaire. Paris: La fabrique éditions.
Maiorana, S.(2010)La importancia de la afectividad en la formación docente universitaria. Congreso Iberoamericano de Educación en Argentina. Disponible en: http:// www.chubut.edu.ar/descargas/secundaria/congreso/ DOCENTES/RLE2571 Maiorana.pdf.

Martínez, (2009). Sistema educativo y didáctica. Madrid: Edición Madrid.

Maslow, A (1998). Hombre autorrealizado: hacia una psicología del ser. Editorial Kairos. Primera edición. Barcelona. ISBN: 9788472452282

May, O. (2001) Pedagogía del afecto: Un amalgamamiento de perspectivas para la educación del colombiano del nuevo milenio. Revista Zona Próxima 2, 30-43.

MClean, Alan. (2003). The motivated school. Great Britain. Ed. P. Chapman.

Mena, I., Vizcarra, R., \& Sepúlveda, G. (2001). Creatividad y planificación de la enseñanza: Estudio en profundidad y medición de impacto de una estrategia para la creatividad en los sistemas educativos. Creatividad y planificación de la enseñanza: Estudio en profundidad $y$ medición de impacto de una estrategia para la creatividad en los sistemas educativos. Santiago: Pontificia Universidad Católica de Chile.

Ministerio de Educación Argentina (2007) disponible en: http:// www.cepal.org/dds/innovacionsocial/e/proyectos/doc/ Proyecto.PrevencionViolencia.Argentina.pdf

Morrison, G. (2005). Educación infantil. Novena edición. Madrid: Pearson Education.

Nielsen, J (2010). Actividades didácticas de motivación en el aula para la enseñanza de inglés como lengua extranjera de los estudiantes de grado undécimo del Colegio Champagnat de Bogotá. Colombia. disponible en: http:// repository.lasalle.edu.co/bitstream/handle/10185/7862/ T26.10\%20N554a.pdf? sequence $=1$

Omart E., Brunetti J., (2009) Conocimiento, afectividad y acción anudados en el aprendizaje ético. Santa Fe, Argentina.

Ortega y Gasset J. (1930) Misión de la Universidad, recuperado en www.esi2.us.es/fabio/mision.pdf.

Páez, D., Echebarría, A; Valencia, J.F; Romo, I; San Juan, C y Vergara, A. (1991). AIDS social representations: Contents and processes. Journal of Community and Applied Psychology, 1, 89-104.

Peláez Peláez, L. M. (2012). "Tipos de aula y ambiente social en el proceso de aprendizaje, en el nivel de educación básica. Estudio realizado en las instituciones educativas escuela Fiscomisional Pedro de Vergara y colegio Fiscomisional Río Cenepa de la ciudad de Gualaquiza, provincia de Morona Santiago, en el año lectivo 2011- 2012" disponible en http://dspace.utpl.edu.ec/handle/123456789/3534 
Peralta J. R (2012) Gestión pedagógica en el aula: clima social escolar, desde la percepción de estudiantes y profesores del séptimo año de educación básica de los centros educativos "República de Colombia y Juan Bautista Vázquez", de la provincia del Azuay, en el año lectivo 2011-2012, Universidad Técnica Particular de Loja, Ecuador.

Piaget, J. (1932): The moral judgment of the child. Translated by M. Worden. New York: Harcourt, Brace, and Word. Disponible en: https://archive.org/stream/ moraljudgmentoft005613mbp\#page/n5/mode/2up

Real Academia de la Lengua Española. (2014). Diccionario de la Real Academia Española. Tomado de: http://www.rae. es/.

Rich, A. (2004). Sangre, pan y poesía. Prosa escogida 1979-1985. Barcelona: Icaria.

Rodríguez-Gómez, G.; Ibarra, M; Gallego-Noche, B; Gómez-Ruiz, M. y Quesada-Serra, V. (2012). La voz del estudiante en la evaluación del aprendizaje: un camino por recorrer en la universidad.

Rodríguez Gómez, G., Ibarra Sáiz, M.S. y Gómez Ruiz, M.A. (2011). e-Autoevaluación en la universidad: un reto para profesores y estudiantes. Revista de Educación. Avance de publicación on-line. DOI:10-4438/1988592X-RE-2010-356-045.

Rodríguez, M. del C. (2011). TIC. Trayectos Interactivos para Compartir. (Tesis doctoral). España, Universidad Nacional de Educación a Distancia (UNED).

Sánchez, (s.f.). El Amor y la Afectividad en el Ser Humano. Tomado de: http://www.rmm.cl/index sub2.php?id contenido $=9453 \&$ id portal $=154 \&$ id + seccion $=859$.

Solís, E; Porlán, R; Rivero, A y Del Pozo, M. (2012). Las concepciones de los profesores de ciencias de secundaria en formación inicial sobre metodología de enseñanza. Revista Española de Pedagogía Vol. 70, No. 253, 495-514.

Touriñán, J. M. (2013). El significado de la función pedagógica y la necesidad de generar principios de acción. Revista española de pedagogía, 71, 29-47.

Valverde, P (2011). 'La motivación y su influencia en el proceso de Enseñanza-Aprendizaje en nivel inicial con los Niños de 3 a 4 años de las escuelas fiscales del Sector sur de la parroquia 9 De mayo del cantón Machala para el año 2010-2011. (Tesis doctoral).

Valdez, D. (2000). Relaciones interpersonales y práctica comunicativa en el contexto escolar. Psicología Educacional. Perspectivas e interrogantes. Buenos Aires: Eudeba.

Vázquez, V. y Escámez, J. (2010) La profesión docente y la ética del cuidado, Revista Electrónica de Investigación
Educativa, (Núm. especial). http://redie.uabc.mx/ contenido/NumEspl/contenido $\wedge /$ erdera.html.

Zúñiga, M. (2010). Relevancia de la sensibilidad pedagógica ante la diversidad educativa. México: Congreso Iberoamericano de Educación.

Maiorana, S. (2010) La importancia de la afectividad en la formación docente universitaria. Congreso Iberoamericano de Educación. Buenos Aires. Argentina. Disponible en: http://www.chubut.edu. ar/descargas/secundaria/congreso/DOCENTES/ RLE2571_Maiorana.pdf

Marí, R. (2013). La actualidad de los lenguajes educativos en la universidad y de su impacto en las prácticas docentes y académicas. Athenea Digital, 13(1), 179-196.

Martínez, (2009). Sistema educativo y didáctica, Edición Madrid.

Maslow, A (1998). Hombre autorrealizado: hacia una psicología del ser. Editorial Kairos. Primera edición. Barcelona.

May, O. (2001) Pedagogía del afecto: Un amalgamamiento de perspectivas para la educación del colombiano del nuevo milenio. Revista Zona Próxima. 2, 30-43.

Ministerio de Educación Argentina (2007) disponible en: http:// www.cepal.org/dds/innovacionsocial/e/proyectos/doc/ Proyecto.PrevencionViolencia.Argentina.pdf

Morejón, Y. y Hernández, Y. (2013) Un arma para el futuro: el uso científico de los medios audiovisuales en la enseñanza de la Literatura. Revista Infociencia. 17(1), 1-12.

Morrison, G. (2005) Educación infantil. Pearson Education.

Mirón, D. M. N. D., Acevedo-Marrero, Á., \& Sánchez-Valdivieso, E. (2014). Estilos de aprendizaje y desempeño académico en Dermatología. Dermatología Revista Mexicana, 58(2), 157-164.

Nielsen, J (2010). Actividades didácticas de motivación en el aula para la enseñanza de inglés como lengua extranjera de los estudiantes de grado undécimo del Colegio Champagnat de Bogotá. Colombia. disponible en: http:// repository.lasalle.edu.co/bitstream/handle/10185/7862/ T26.10\%20N554a.pdf?sequence $=$.

Omart E., Brunetti J., (2009) Conocimiento, afectividad y acción anudados en el aprendizaje ético. Santa Fe, Argentina.

Ortega y Gasset J. (1930) Misión de la Universidad, recuperado 
en www.esi2.us.es/fabio/mision.pdf.

Páez, D., Echebarría, A:, Valencia, J.F., Romo, I, San Juan, C., y Vergara, A. (1991). AIDS social representations: Contents and processes. Journal of Community and Applied .Psychology, 1, 89-104.

Peláez Peláez, L. M. (2012). "Tipos de aula y ambiente social en el proceso de aprendizaje, en el nivel de educación básica. Estudio realizado en las instituciones educativas escuela Fiscomisional Pedro de Vergara y colegio Fiscomisional Río Cenepa de la ciudad de Gualaquiza, provincia de Morona Santiago, en el año lectivo 2011- 2012" disponible en: http://dspace.utpl.edu.ec/handle/123456789/3534.

Peralta J. R (2012) Gestión pedagógica en el aula: clima social escolar, desde la percepción de estudiantes y profesores del séptimo año de educación básica de los centros educativos "República de Colombia y Juan Bautista Vázquez", de la provincia del Azuay, en el año lectivo 2011-2012, Universidad Técnica Particular de Loja, Ecuador.

Piaget, J. (1932): The moral judgment of the child. Translated by M. Worden. New York: Harcourt, Brace, and Word. Disponible en: https://archive.org/stream/ moraljudgmentoft005613mbp\#page/n5/mode/2up.

Quilambaqui, J. (2011). 'Análisis de la inteligencia emocional en el desempeño profesional del personal de la unidad educativa Mario Rizzini de la ciudad de Cuenca. Tesis. Universidad Politécnica Salesiana del Ecuador. Ecuador.

Rafael-Linares, A. (2009). Desarrollo cognitivo: Las teorías de Piaget y Vygotsky. Recuperado de: http://www. paidopsiquiatria.cat/files/Teorias_desarrollo_cognitivo. pdf.

Rodríguez-Gómez, G., Ibarra-Sáiz, M. S., Gallego-Noche, B., Gómez-Ruiz, M. Á., \& Quesada-Serra, V. (2012). La voz del estudiante en la evaluación del aprendizaje: un camino por recorrer en la universidad. Relieve, 18(2), 133-153.

Rodríguez Gómez, G., Sáiz, I., Soledad, M., \& Gómez Ruiz, M. Á. (2011). e-Autoevaluación en la universidad: un reto para profesores y estudiantes. Revista de Educación, $356,401-430$.
Rodríguez, M. del C. (2011). Trayectos Interactivos para Compartir. Argentina. Disponible en: http://docplayer. es/17086288-T-i-c-trayectos-interactivos-para-compartir.html.

Sánchez, (s.f.). El Amor y La Afectividad en El Ser Humano. http://www.rmm.cl/index sub2.php?id contenido $=9453 \& i d$ portal $=154 \&$ id + seccion $=859$.

Ramírez, E. S., Ariza, R. P., García, A. R., \& Del Pozo, R. M. (2012). Las concepciones de los profesores de ciencias de secundaria en formación inicial sobre metodología de enseñanza. Revista Española de Pedagogía, 495-514.

Touriñán, J. M. (2013). El significado de la función pedagógica y la necesidad de generar principios de acción. Revista española de pedagogía, 71 (254), 29-47.

Valdez, D. (2000). Relaciones interpersonales y práctica comunicativa en el contexto escolar. C. Chardón (Comp) Perspectivas e interrogantes en Psicología Educacional. Buenos Aires: Eudeba.

Vázquez, V. y Escámez, J. (2010) Vázquez Verdera, V., \& Escámez Sánchez, J. (2010). La profesión docente y la ética del cuidado. Revista electrónica de investigación educativa, 12(SPE.), 1-17.

Vygotski, L. S. (1984). Aprendizaje y desarrollo intelectual en la edad escolar. Infancia y aprendizaje, 7(27-28), 105-116.

Zúñiga, M. (2010). Relevancia de la sensibilidad pedagógica ante la diversidad educativa. Congreso Iberoamericano de Educación. México. Disponible en: http://www.adeepra. org.ar/congresos/Congreso\%20IBEROAMERICANO/ EDUCINCLUSIVA/RLE2370_Zuniga.pdf. 